\title{
BMJ Open Liver fat content is independently associated with microalbuminuria in a normotensive, euglycaemic Chinese population: a community-based, cross- sectional study
}

Xiaoming Li, ${ }^{1,2}$ Mingfeng Xia, ${ }^{1}$ Hui Ma, ${ }^{1}$ Yu Hu (D) , ${ }^{1}$ Hongmei Yan, ${ }^{1}$ Wanyuan $\mathrm{He},{ }^{3}$ Huandong Lin, ${ }^{1}$ Nai Qing Zhao, ${ }^{4}$ Jian Gao, ${ }^{5}$ Xin Gao (D) ${ }^{1}$

To cite: Li X, Xia M, Ma H, et al. Liver fat content is independently associated with microalbuminuria in a normotensive, euglycaemic Chinese population: a community-based, crosssectional study. BMJ Open 2021;11:e044237. doi:10.1136/ bmjopen-2020-044237

- Prepublication history and supplemental material for this paper is available online. To view these files, please visit the journal online (http://dx.doi. org/10.1136/bmjopen-2020 044237).

Received 04 September 2020 Accepted 02 June 2021

Check for updates

(c) Author(s) (or their employer(s)) 2021. Re-use permitted under CC BY-NC. No commercial re-use. See rights and permissions. Published by BMJ.

For numbered affiliations see end of article.

Correspondence to

Dr Xin Gao;

zhongshan_endo@126.com

\section{ABSTRACT}

Objective Non-alcoholic fatty liver disease (NAFLD) is associated with microalbuminuria (MA) in patients with diabetes/pre-diabetes. Whether this association is mediated by blood glucose and blood pressure (BP) remains unclear. This study investigated whether liver fat content (LFC) was associated with MA in a normotensive and non-diabetic population.

Design A cross-sectional substudy.

Settings LFC was determined from the hepatic/renal echogenicity ratio at ultrasound. MA was defined as an albumin-to-creatinine ratio (ACR) of 30-300 $\mu \mathrm{g} / \mathrm{mg}$ (earlymorning urine sample). Multivariable logistic regression and receiver operating characteristic (ROC) curve analyses were used to evaluate LFC as a predictor of MA.

Participants Between May 2010 and June 2011, this cross-sectional, community-based study enrolled residents from Shanghai (China), aged $\geq 40$ years and with normal glucose tolerance and $\mathrm{BP}$.

Results A total of 550 residents (median age, 57 years; 174 men) were enrolled and stratified according to LFC quartiles. ACR $(p<0.001)$ and MA prevalence $(p=0.012)$ increased across the LFC quartiles. Multivariable logistic regression showed that the OR for MA (per SD increase in LFC) was 1.840 (95\% Cl 1.173 to $2.887, p=0.008)$ after adjustment for potential confounders including age, gender, waist-hip ratio, blood urea nitrogen, systolic and diastolic BP, fasting blood glucose, postprandial glucose, low-density lipoprotein-cholesterol, triglycerides, highdensity lipoprotein-cholesterol, total cholesterol, estimated glomerular filtration rate and lipid-lowering drugs. The ROC analysis revealed that the optimal LFC cut-off value for predicting MA was $6.82 \%$.

Conclusion LFC is independently associated with MA in normotensive, euglycaemic middle-aged and elderly Chinese individuals. Screening for MA in people with NAFLD might facilitate early intervention to minimise kidney disease risk.

\section{INTRODUCTION}

Non-alcoholic fatty liver disease (NAFLD) is an important global cause of chronic liver disease. ${ }^{1}$ NAFLD's global prevalence is

\section{Strengths and limitations of this study}

- This study included a relatively large number of individuals and used a quantitative method based on ultrasound, an inexpensive and readily available technology.

- This study was cross sectional, and causality could not be inferred.

- The diagnosis of non-alcoholic fatty liver disease was based on ultrasound findings and was not confirmed by biopsy; microalbuminuria was based on an early-morning urine sample and was not confirmed by 24-hour urine sampling.

around 24\%, with South America, the Middle East and Asia having the highest rates. ${ }^{23}$ The manifestations of NALFD range from simple steatosis to non-alcoholic steatohepatitis, hepatic fibrosis, liver cirrhosis and hepatocellular carcinoma. ${ }^{14}$ NAFLD is associated with an increased risk of cardiovascular disease $(\mathrm{CVD})^{5}$ and with components of the metabolic syndrome (MetS), including visceral obesity, dyslipidaemia, insulin resistance and type 2 diabetes mellitus (T2DM). ${ }^{15-7}$ Still, NAFLD can occur without T2DM or MetS, which can be protective for CVD in some cases. ${ }^{8}$ Therefore, the mechanisms underlying the associations of NAFLD with MetS remain incompletely understood but may involve a complex interplay of metabolic factors and inflammatory cytokines. ${ }^{9}$

Microalbuminuria (MA) is a urinary albumin excretion of $30-300 \mathrm{mg} / 24$ hours and is an early marker of renal endothelial dysfunction and glomerular injury. ${ }^{10} 11$ In apparently healthy individuals, an accelerated change in MA is associated with the development of T2DM and hypertension. ${ }^{12}$ Importantly, MA is associated with all-cause 
mortality in apparently healthy individuals. ${ }^{13} 14$ MA is associated with several cardiovascular risk factors in nondiabetic individuals, including gender, systolic blood pressure (SBP), total cholesterol (TC), smoking and body mass index (BMI).${ }^{15}{ }^{16}$ Since components of MetS are associated with MA, ${ }^{17}$ there has been interest in establishing whether NAFLD is also related to MA. NAFLD is associated with MA in patients with pre-diabetes and T2DM. ${ }^{18}$ Furthermore, studies in non-diabetic and diabetic patients have suggested that renal dysfunction is associated with the severity of NAFLD and liver fibrosis. ${ }^{19-23}$ Sun $e t a l^{4}$ showed that liver fibrosis is independently associated with early kidney dysfunction (shown by MA and an estimated glomerular filtration rate (eGFR) $>60 \mathrm{~mL} /$ $\min / 1.73 \mathrm{~m}^{2}$ ) in patients with NAFLD proven by biopsy. NAFLD may also be associated with low-grade albuminuria, ${ }^{25}$ and some studies have identified NAFLD as a risk factor for diabetic nephropathy in patients with T1DM and T2DM. ${ }^{26}{ }^{27}$ A genetic basis could also be involved in NAFLD and chronic kidney disease (CKD), and a study showed that a specific genetic change in patients with NAFLD but normal liver enzyme levels were at higher risk of early glomerular and tubular damage. ${ }^{28}$ However, it remains unclear whether the association between NAFLD and MA is mediated by factors such as blood glucose level and blood pressure (BP). Interestingly, a recent metaanalysis indicated that NAFLD increased the risk of albuminuria in people without diabetes but not those with diabetes. ${ }^{29}$ Confirming an association between NAFLD and MA in normotensive and euglycaemic people would have important implications, suggesting that regular screening for MA to detect early-stage renal damage in patients with NAFLD would facilitate timely intervention to improve renal outcomes.

To our knowledge, no previous reports have evaluated whether NAFLD increases the risk of MA in people with normal BP and blood glucose levels. Therefore, this study aimed to investigate whether liver fat content (LFC) was associated with MA in a normotensive and non-diabetic population of middle-aged and elderly Chinese people in whom confounding factors such as blood glucose level and BP were optimally controlled. It was anticipated that the findings might have important implications with regard to the use of early preventive and therapeutic approaches.

\section{MATERIAL AND METHODS Study population}

This cross-sectional substudy included participants of the Changfeng Study, which was a community-based cohort study of chronic diseases among middle-aged and elderly people in Shanghai, China. ${ }^{30}$ Residents aged $\geq 40$ years were consecutively screened for inclusion between May 2010 and June 2011. The exclusion criteria were (1) abnormal glucose level (impaired fasting glucose or impaired glucose tolerance) or T2DM (diagnosed diabetes or newly diagnosed diabetes) and hypertension;
(2) incomplete data; (3) alcohol misuse; (4) viral hepatitis; and (5) urinary system diseases or hepatic dysfunction (defined as $>1.5$-fold elevation of alanine aminotransferase (ALT), aspartate aminotransferase (AST) or direct bilirubin); or (6) urinary tract infection and haematuria via routine urine test. Hypertension was defined as published in the Seventh Report of the Joint National Committee on Prevention, Detection, Evaluation, and Treatment of High Blood Pressure. ${ }^{31}$ The diagnosis of diabetes mellitus was based on the 2010 criteria by the American Diabetes Association. ${ }^{32} \mathrm{MetS}$ was defined according to the joint interim statement. ${ }^{33}$ The study protocol was approved by the Research Ethics Committee of Shanghai Health Bureau. Written informed consent was obtained from all participants. Physical examinations, laboratory assessments and liver ultrasound scans were performed on the same morning for each individual at Changfeng Community Hospital.

\section{LFC measurement}

All 550 participants underwent hepatic ultrasound (Logiq P5 scanner with a $4 \mathrm{MHz}$ probe; GE Healthcare, Milwaukee, Wisconsin, USA) performed by an experienced radiologist blinded to the participants' details. Ultrasound images of the liver and right kidney were obtained as previously described. ${ }^{34}$ The echo intensities of the liver and kidney were measured using Image V.1.41o (National Institutes of Health, Bethesda, Maryland, USA), with standardisation using a tissue-mimicking phantom. LFC was calculated from the hepatic/renal echogenicity ratio. ${ }^{34}$ Repeated measurements on 102 subjects randomly selected from the 550 participants yielded an intraclass correlation coefficient of $95 \%$.

\section{Detection of MA}

The albumin-to-creatinine ratio (ACR) in an earlymorning urine sample was measured using an immunonephelometric method. MA was defined as an ACR of $30-300 \mu \mathrm{g} / \mathrm{mg}^{35}$

\section{Evaluation of other parameters}

Trained nurses interviewed all participants using a standardised questionnaire to obtain information regarding their medical history and health-related behaviours. Weight and height were recorded, and BMI was calculated as weight divided by height squared $\left(\mathrm{kg} / \mathrm{m}^{2}\right)$. Waist circumference was measured midway between the lowest rib margin and the iliac crest in the standing position. Resting BP was measured three times, and the mean value was used for the analyses. Blood samples were obtained after a fasting period $\geq 10$ hours. TC, highdensity lipoprotein-cholesterol (HDL-c), triglycerides (TG), blood urea nitrogen (BUN), creatinine and liver enzymes were measured using a 7600 Automatic Biochemical Analyser (Hitachi, Tokyo, Japan). Low-density lipoprotein-cholesterol (LDL-c) was calculated by the Friedewald equation. Fasting blood glucose (FBG) and 2-hour glucose levels after a $75 \mathrm{~g}$ oral glucose challenge 
(postprandial glucose, PPG) were measured using the glucose oxidase method. eGFR was calculated Chronic Kidney Disease Epidemiology Collaboration (CKD-EPI) equations. ${ }^{36}$

\section{Statistical analysis}

Continuous variables are presented as mean \pm SD if normally distributed or median (IQR) if non-normally distributed. Categorical variables are expressed as numbers (percentages). Skewed variables were logarithmically transformed before analysis, and all were normally distributed after $\log$ transformation. To evaluate a potential correlation between metabolic parameters and LFC, subjects were stratified according to LFC quartiles: the median (IQR) of each LFC quartile was $2.09 \%$ $(0 \%-3.07 \%), 4.15 \% \quad(3.09 \%-5.20 \%), 6.88 \% \quad(5.25 \%-$ $8.72 \%)$ and $14.28 \%(8.75 \%-48.02 \%)$. Analysis of variance was used to analyse the continuous variables among the four groups. The least significant diffierence (LSD) post hoc analysis was used for pairwise comparisons. The t-test for independent samples was used for the analysis of continuous variables between two groups. The $\chi^{2}$ and Fisher's exact tests were used to compare categorical variables among groups. Multivariable logistic regression analysis was performed to test the association between LFC and MA after adjustments for potential confounders in an enter method. The results are expressed as OR per SD with 95\% CIs. Model 1 was adjusted for age and gender. Model 2 was additionally adjusted for waist-hip ratio (WHR) and BUN. Model 3 was the same as model 2, except that WHR was replaced by waist and hip circumferences. Model 4 was model 2 additionally adjusted for SBP, diastolic BP (DBP), FBG, PPG, LDL-c, TG, HDL-c, TC, eGFR and lipid-lowering drugs. Model 5 was the same as model 4, except that WHR was replaced by waist and hip circumferences. Receiver operating characteristic (ROC) curve analysis was used to determine the optimal cut-off LFC value for identifying people with MA, which was calculated using the Youden index. SPSS V.17.0 for Windows (SPSS) was used for analysis. All statistical tests were two-tailed, and $\mathrm{p}$ values $<0.10$ were considered statistically significant.

\section{Patient and public involvement}

How were the development of the research question and outcome measures informed by patients' priorities, experiences and preferences?

The study was a cross-sectional substudy including participants of the Changfeng Study. Residents aged $\geq 40$ years were consecutively screened for inclusion between May 2010 and June 2011. The Shanghai Changfeng Study-a community-based prospective cohort study of chronic diseases among middle-aged and elderly-was designed in collaboration with principal investigators of the Rotterdam Study, making the Shanghai Changfeng Study a comparative study to the Rotterdam Study. ${ }^{30}$ The study was designed to study the aetiology and prognosis of chronic diseases. A questionnaire has been prepared based on the questionnaire of the Rotterdam Study. ${ }^{37}$ We modified the questionnaire according to the lifestyle, customs and characteristics of people in Shanghai. The whole questionnaire includes 25 domains and 414 items. Besides demographic data and lifestyle factors, the domains are classified according to specific diseases.

How did you involve patients in the design of this study?

The study population comprises those who are 40 years old or older in the Changfeng community of the Putuo District in Shanghai, China, between May 2010 and June 2011. In fact, they are the residents, not the patients.

Were patients involved in the recruitment to and conduct of the study?

Yes, they are. The initial study population comprises those who are 40 years old or older in the Changfeng community of the Putuo District in Shanghai, China, no matter they were healthy or not. However, the people involved in this study were relatively 'healthy' populations with normal glucose tolerance and BP.

How will the results be disseminated to study participants?

We printed out the results of last week's physical examination in the form of a report, sent them to the residents and gave them medical advice every week.

\section{RESULTS}

\section{Clinical characteristics of the study participants}

Among 2225 residents screened, 1675 were excluded due to abnormal glucose level or BP $(\mathrm{n}=1592)$, incomplete data $(n=28)$, alcohol misuse $(n=25)$, viral hepatitis $(n=26)$ or other diseases such as urinary tract infection or hepatic dysfunction (defined as $>1.5$-fold elevation of ALT, AST or direct bilirubin) $(\mathrm{n}=4)$. Therefore, 550 subjects $(174$ men, 31.6\%) with a median age of $57(53-64)$ years and a median BMI of 22.59 (20.79-24.61) kg/m² were included in the final analyses. Table 1 shows the characteristics of the study participants. The prevalence of dyslipidaemia, MetS and MA was $35.82 \%, 11.45 \%$ and $2.37 \%$, respectively.

\section{Clinical characteristics of the study participants stratified by LFC quartiles}

Of the 2225 participants, 2088 were assessed for LFC. The range of the LFC of all people was $0 \%-49.94 \%$ (IQR $3.26 \%-14.24 \%$ ) (online supplemental figure 1A). The LFC range of the excluded individuals was $0 \%-49.94 \%$ (IQR 3.32\%-15.99\%) (online supplemental figure 1B). The LFC range of the study population was $0 \%-48.02 \%$ (IQR 3.09\%-8.76\%) (online supplemental figure 1C). The LFC distribution of the included individuals (range $0 \%-48.02 \%$ ) was non-Gaussian and positively skewed with a tail extending to higher values. According to the criteria for steatosis diagnosis by quantitative ultrasonography, $23.09 \%$ of the subjects $(127 / 550)$ had hepatic steatosis. The clinical characteristics of the participants stratified by LFC quartiles are presented in table 1 . There were significant increases in the waist, hip, BMI, WHR, SBP, DBP, FBG, PPG, TC, LDL-c, TG, ALT, ACR and MA prevalence 


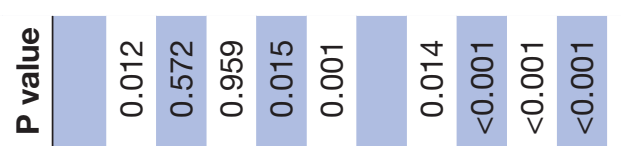

웅 ○े @

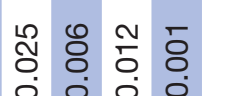

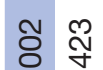
i

ণั่

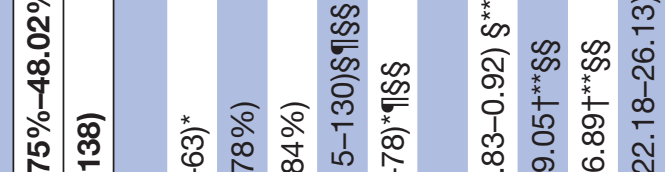
N

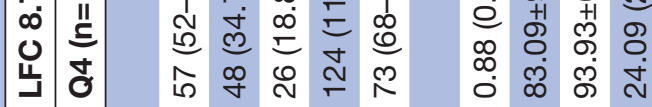

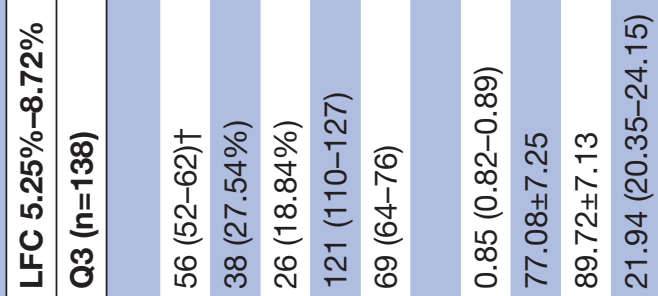

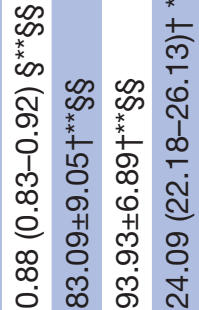

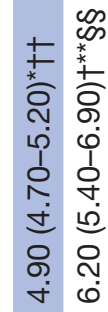
एं เก

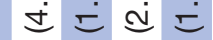

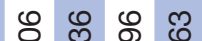
ĭ

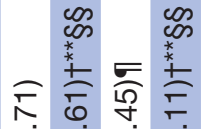

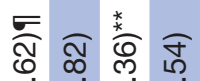

i.

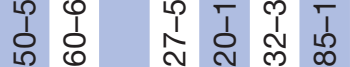

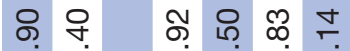

更
ذ̇
$+$

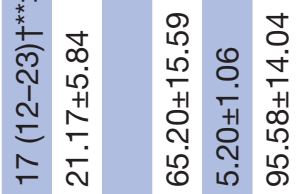

$\infty$

$\stackrel{\sqrt[0]{0}}{+\infty}$

ㅇำ

ㅇํㅇ ㅇํㅇ

¿

in vi ne

๑ $\infty \frac{N}{N}$
ฉั่

นิ

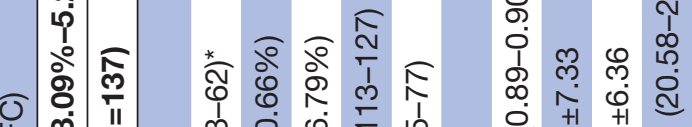

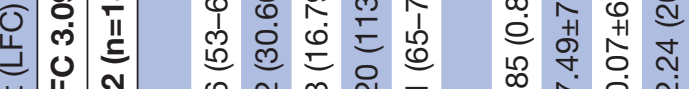

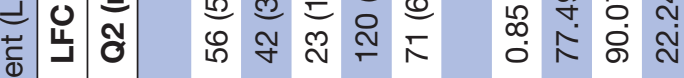
خे

苋

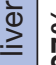

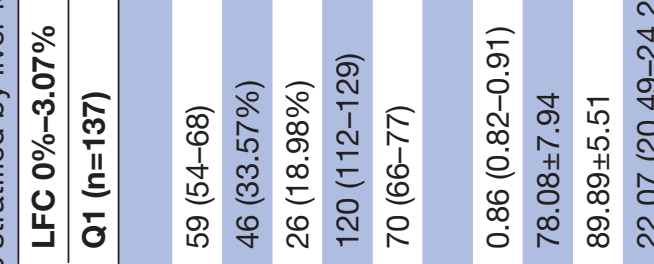

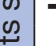

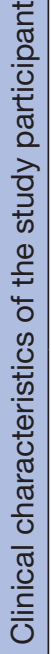

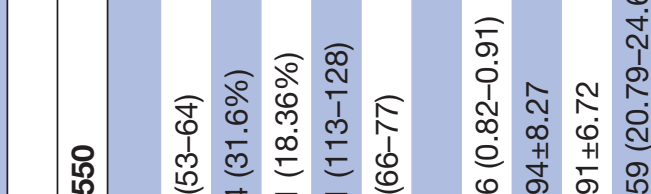

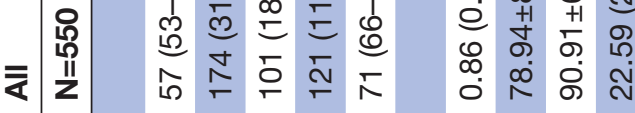

政

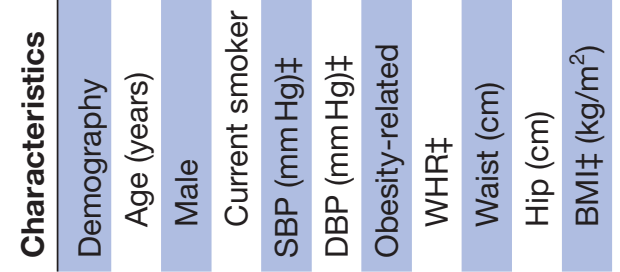

है

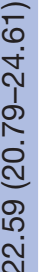

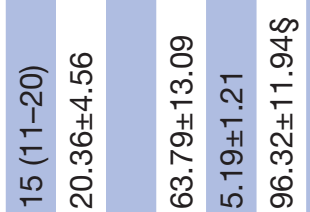

蒙

๙

守 $\sqrt{2}$

잉

ㅇำ ले

मे ले

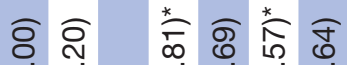

एं

d

¿̇ $\dot{ \pm}=\mathbb{I}$

인 กำ

นึ

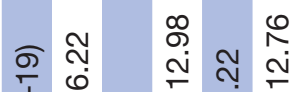

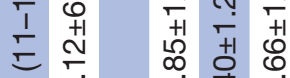

ஏ

$\overparen{\infty}$

jo

○)

¿

융

मं

บ

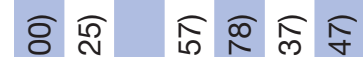

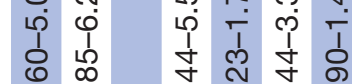

¿

$\infty$

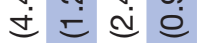

ก

४

チ

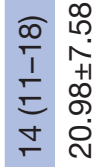

হ

$\stackrel{5}{\infty}$

ก)

ต

ल

으늠

ก

(ब) ले

$\infty$

กำ ฮิ

மं

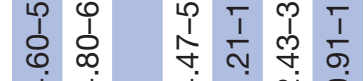

过过

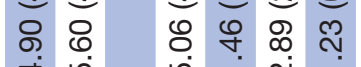

क サ

으

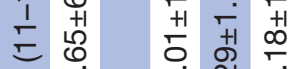

苚

ํ. ले

@ ल

เก ญे

เึ่ กิ คี

ণั

ติ

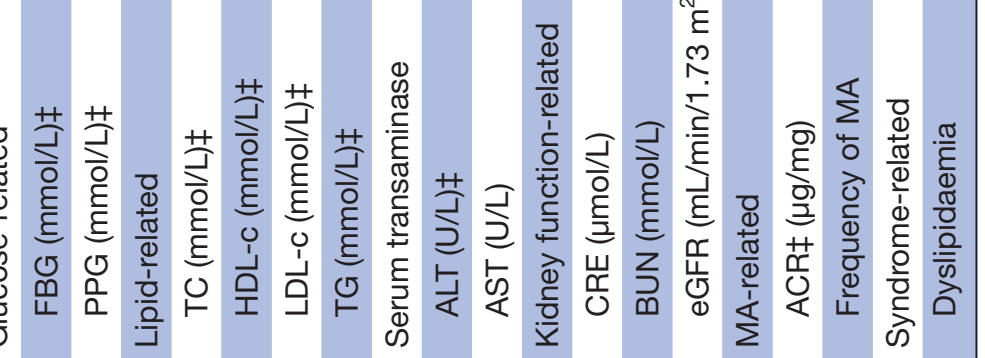

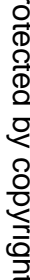




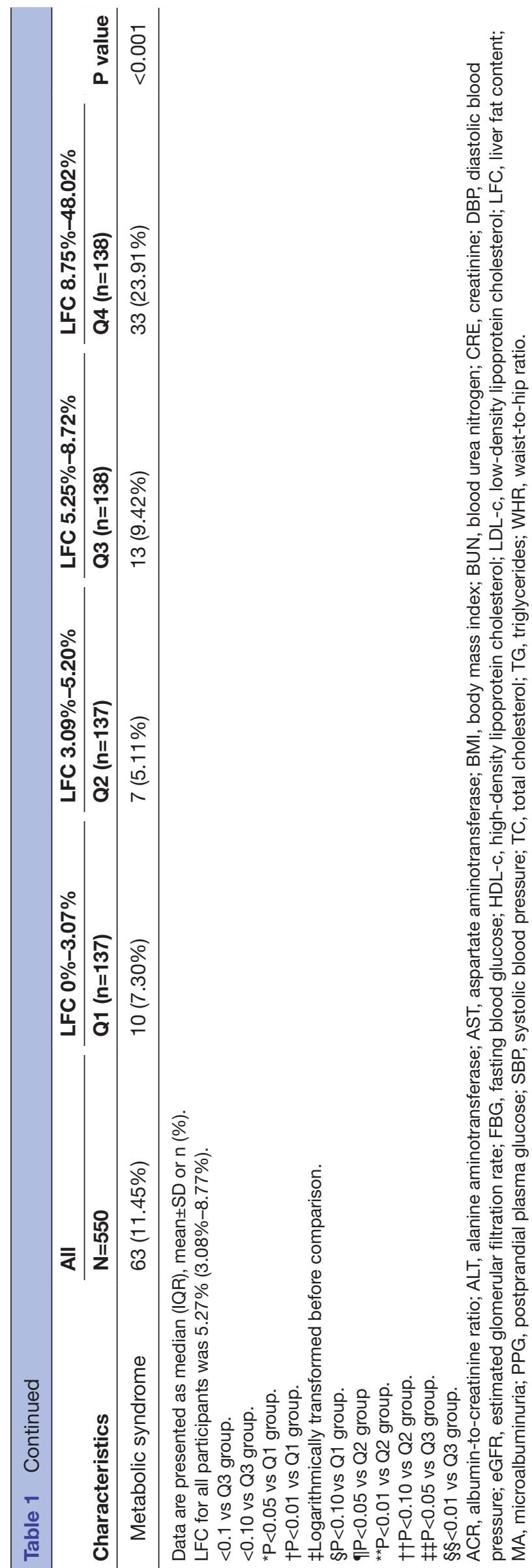

across LFC quartiles (all P-for-trend $<0.10$ ). Age and HDL-c decreased across the quartiles $(p=0.012$ and $\mathrm{p}=0.006$, respectively). eGFR, CRE, BUN, AST, smoking status and gender did not vary across LFC quartiles.

\section{Clinical characteristics of the study participants stratified by} urinary albumin excretion

Table 2 shows the characteristics of patients stratified by urine albumin status. Patients with albuminuria had higher waist $(\mathrm{p}=0.067)$, WHR $(\mathrm{p}=0.047)$, BMI $(\mathrm{p}=0.080)$, LDL-c $(p=0.090)$, AST $(p=0.031)$ and BUN $(p=0.026)$ than those without albuminuria, whereas gender, age, LFC, SBP, DBP, hip, FBG, PPG, TC, HDL-c, TG, ALT, CRE, smoking status, prevalence of dyslipidaemia and prevalence of MetS did not differ between groups.

\section{Multivariable logistic regression analysis of the association between LFC and MA}

In order to examine the relationship between LFC and MA, logistic regression was performed with adjustment for potential confounders in an enter manner (table 3). The association between LFC and MA was significant without adjustment (OR 1.549; 95\% CI 1.099 to 2.184; $\mathrm{p}=0.013$ ) and remained significant after adjustment for age and sex (model 1: OR 1.669; 95\% CI 1.161 to 2.399; $\mathrm{p}=0.006$ ). The association remained significant after further adjustment for WHR (or for waist and hip circumferences, model 3; or for BMI, model 4) and BUN (model 2: OR 1.706; 95\% CI 1.167 to 2.496; $\mathrm{p}=0.006$; model 3: OR $1.839 ; 95 \%$ CI 1.172 to 2.886 ; $\mathrm{p}=0.008$; model 4: OR $1.617 ; 95 \%$ CI 1.076 to 2.431; $\mathrm{p}=0.021$ ), for WHR (or for waist and hip circumferences, model 6; or for BMI, model 7), BUN, SBP, DBP, FBG, PBG, LDL-c, TG, HDL-c, TC, eGFR and lipid-lowering drugs (model 5: OR 1.840; $95 \%$ CI 1.173 to 2.887; $\mathrm{p}=0.008$; model 6 : OR $1.776 ; 95 \%$ CI 1.108 to $2.847 ; \mathrm{p}=0.017$; model 7 : OR $1.705 ; 95 \%$ CI 1.064 to $2.730 ; \mathrm{p}=0.026$ ).

ROC curve analysis of the utility of LFC in the prediction of MA The ability of LFC to predict MA was evaluated by ROC curve analysis (figure 1). The area under the curve was 0.738 (95\% CI 0.594 to $0.882, \mathrm{p}=0.003$ ). The optimal LFC cut-off value for the diagnosis of MA was $6.82 \%$. The sensitivity and specificity of this LFC cut-off value for diagnosing MA were $84.6 \%$ and $63.0 \%$, respectively.

\section{DISCUSSION}

A notable finding of this study was that the prevalence of hepatic steatosis (measured by quantitative ultrasound) was $23.09 \%$ in this normotensive and euglycaemic population. Importantly, LFC was significantly associated with MA in this population, even after adjustment for possible confounders such as cardiovascular risk factors and MetS components. In addition, an optimal LFC cut-off value of $6.82 \%$ was able to predict MA with a sensitivity of $84.6 \%$ and specificity of $63.0 \%$. Even though the prevalence of MA in normoglycaemic, normotensive patients 
Table 2 Clinical characteristics of the study participants, stratified by albuminuria status

\begin{tabular}{|c|c|c|c|}
\hline \multirow{2}{*}{ Characteristics } & \multicolumn{2}{|l|}{ Microalbuminuria } & \multirow[b]{2}{*}{$P$ value } \\
\hline & No $(n=537)$ & Yes $(n=13)$ & \\
\hline \multicolumn{4}{|l|}{ Demography } \\
\hline Age (years) & 57 (53-64) & $62(54-68)$ & 0.301 \\
\hline Male & $172(32.03 \%)$ & $2(15.38 \%)$ & 0.363 \\
\hline Current smoker & 98 (18.25\%) & $2(15.38 \%)$ & 1.000 \\
\hline LFC $(\%)^{\star}$ & $5.18(3.06-8.65)$ & $10.30(7.18-16.64)$ & 0.400 \\
\hline $\mathrm{SBP}(\mathrm{mm} \mathrm{Hg})^{*}$ & $120(113-128)$ & $127(120-130)$ & 0.388 \\
\hline $\mathrm{DBP}(\mathrm{mm} \mathrm{Hg})^{*}$ & $71(66-77)$ & $76(67-79)$ & 0.695 \\
\hline \multicolumn{4}{|l|}{ Obesity-related } \\
\hline $\mathrm{WHR}^{\star}$ & $0.86(0.82-0.90)$ & $0.90(0.88-0.95)$ & 0.047 \\
\hline Waist $(\mathrm{cm})^{\star}$ & $78.80(73.00-83.50)$ & $83.00(77.00-86.75)$ & 0.067 \\
\hline $\operatorname{Hip}(\mathrm{cm})^{*}$ & $91.00(87.00-95.00)$ & $90.00(87.75-97.00)$ & 0.660 \\
\hline $\operatorname{BMI}\left(\mathrm{kg} / \mathrm{m}^{2}\right)^{\star}$ & 22.53 (20.78-24.59) & $24.16(22.39-25.30)$ & 0.080 \\
\hline \multicolumn{4}{|l|}{ Glucose-related } \\
\hline $\mathrm{FBG}(\mathrm{mmol} / \mathrm{L})^{\star}$ & $4.80(4.60-5.00)$ & $4.90(4.80-5.30)$ & 0.178 \\
\hline $\mathrm{PPG}(\mathrm{mmol} / \mathrm{L})^{*}$ & $5.60(4.80-6.50)$ & $5.60(4.80-6.50)$ & 0.942 \\
\hline \multicolumn{4}{|l|}{ Lipid-related } \\
\hline $\mathrm{TC}(\mathrm{mmol} / \mathrm{L})^{\star}$ & $5.05(4.47-5.64)$ & $5.68(5.05-5.84)$ & 0.111 \\
\hline $\mathrm{HDL}-\mathrm{c}(\mathrm{mmol} / \mathrm{L})^{\star}$ & $1.46(1.21-1.74)$ & $1.49(1.17-1.71)$ & 0.798 \\
\hline LDL-c $(\mathrm{mmol} / \mathrm{L})^{*}$ & $2.89(2.43-3.43)$ & $3.38(2.83-3.78)$ & 0.090 \\
\hline $\mathrm{TG}(\mathrm{mmol} / \mathrm{L})^{\star}$ & $1.23(0.91-1.71)$ & $1.41(1.06-1.72)$ & 0.438 \\
\hline \multicolumn{4}{|l|}{ Serum transaminase } \\
\hline $\mathrm{ALT}(\mathrm{U} / \mathrm{L})^{\star}$ & $15(11-19)$ & $17(13-26)$ & 0.127 \\
\hline AST $(\mathrm{U} / \mathrm{L})^{*}$ & $19(17-22)$ & $21(20-25)$ & 0.031 \\
\hline \multicolumn{4}{|l|}{ Kidney function-related } \\
\hline CRE $(\mu \mathrm{mol} / \mathrm{L})^{*}$ & $63(54-74)$ & $63(52-74)$ & 0.576 \\
\hline $\mathrm{BUN}(\mathrm{mmol} / \mathrm{L})^{*}$ & $5.20(4.50-6.00)$ & $5.80(5.00-7.10)$ & 0.026 \\
\hline eGFR $\left(\mathrm{mL} / \mathrm{min} / 1.73 \mathrm{~m}^{2}\right)$ & $95.26 \pm 13.49$ & $91.73 \pm 12.74$ & 0.351 \\
\hline \multicolumn{4}{|l|}{ Syndrome-related } \\
\hline Dyslipidaemia (\%) & $192(35.75 \%)$ & $5(38.46 \%)$ & 0.779 \\
\hline Metabolic syndrome (\%) & $60(11.17 \%)$ & $3(23.08 \%)$ & 0.167 \\
\hline
\end{tabular}

Data are presented as median (IQR) or $\mathrm{n}(\%)$.

*Logarithmically transformed before comparison.

ALT, alanine aminotransferase; AST, aspartate aminotransferase; BMI, body mass index; BUN, blood urea nitrogen; CRE, creatinine; DBP, diastolic blood pressure; eGFR, estimated glomerular filtration rate; FBG, fasting blood glucose; HDL-c, high-density lipoprotein cholesterol; LDL-c, low-density lipoprotein cholesterol; LFC, liver fat content; PPG, postprandial plasma glucose; SBP, systolic blood pressure; TC, total cholesterol; TG, triglycerides; WHR, waist-to-hip ratio.

with NAFLD was only $2.4 \%(13 / 550)$, the prevalence of NAFLD was $23 \%$. Since a test for microalbumin costs only $30 \mathrm{RMB}$ in China (less than $\$ 5$ ), screening for MA in apparently 'healthy' people with NAFLD might help identify those at risk of renal damage, allowing early intervention to minimise the long-term CKD risk.

NAFLD frequency in normotensive and euglycaemic people aged $\geq 40$ years was $23.09 \%$, indicating that NAFLD was prevalent in this relatively and apparently healthy population. Although the prevalence of NAFLD in our study was lower than that reported previously $(28.6 \%),{ }^{38}$ this is likely due to differences in the enrolled cohorts between the previous study (the general Hong Kong Chinese population, among whom $5.2 \%$ had T2DM) and our study (normotensive and non-diabetic participants). In addition, the prevalence of MetS was not different between subjects with or without MA, and there was no clear dose-dependent relationship between LFC and MetS. It may be because this study excluded the individuals with elevated glucose levels 
Table 3 Multivariable logistic regression analysis of the association between liver fat content and presence of microalbuminuria

\begin{tabular}{rlll}
\hline Model & OR per SD of LFC & $\mathbf{9 5 \%} \mathbf{C l}$ & P value \\
\hline $\begin{array}{r}\text { Unadjusted } \\
\text { Adjusted }\end{array}$ & 1.549 & 1.099 to 2.184 & 0.013 \\
\hline Model 1 & 1.669 & 1.161 to 2.399 & 0.006 \\
\hline Model 2 & 1.706 & 1.167 to 2.496 & 0.006 \\
\hline Model 3 & 1.839 & 1.172 to 2.886 & 0.008 \\
\hline Model 4 & 1.617 & 1.076 to 2.431 & 0.021 \\
\hline Model 5 & 1.840 & 1.173 to 2.887 & 0.008 \\
\hline Model 6 & 1.776 & 1.108 to 2.847 & 0.017 \\
\hline Model 7 & 1.705 & 1.064 to 2.730 & 0.026 \\
\hline
\end{tabular}

Model 1: Adjusted for age and gender.

Model 2: Adjusted for age, gender, waist-hip ratio and blood urea nitrogen.

Model 3: Adjusted for age, gender, waist, hip and blood urea nitrogen.

Model 4: Adjusted for age, gender, body mass index and blood urea nitrogen.

Model 5: Adjusted for age, gender, waist-hip ratio, blood urea nitrogen, systolic blood pressure, diastolic blood pressure, fasting blood glucose, postprandial glucose, low-density lipoprotein cholesterol, triglycerides, high-density lipoprotein cholesterol, total cholesterol, eGFR and lipid-lowering drug usage.

Model 6: Adjusted for age, gender, waist, hip, blood urea nitrogen, systolic blood pressure, diastolic blood pressure, fasting blood glucose, postprandial glucose, low-density lipoprotein cholesterol, triglycerides, high-density lipoprotein cholesterol, total cholesterol, eGFR and lipid-lowering drug usage.

Model 7: Adjusted for age, gender, body mass index, blood urea nitrogen, systolic blood pressure, diastolic blood pressure, fasting blood glucose, postprandial glucose, low-density lipoprotein cholesterol, triglycerides, high-density lipoprotein cholesterol, total cholesterol, eGFR and lipid-lowering drug usage.

eGFR, estimated glomerular filtration rate; LFC, liver fat content.

and hypertension, leaving mostly metabolically relatively healthy subjects. ${ }^{39}$

Previous studies have reported that NAFLD is associated with MA in patients with pre-diabetes and $\mathrm{T} 2 \mathrm{DM}^{18}$ and low-grade albuminuria in Chinese adults. ${ }^{25}$ Moreover, the severity of liver fibrosis was associated with MA in non-diabetic patients. ${ }^{19}$ NAFLD has also been reported as a risk factor for diabetic nephropathy. ${ }^{26}{ }^{27}$ Our finding that LFC was associated with MA after adjustment for multiple confounding factors is consistent with these previous investigations. However, a recent study reported that advanced liver fibrosis, not steatosis, was independently associated with albuminuria in patients with T2DM. ${ }^{20}$ This inconsistency may result from differences between the study populations and diagnostic tools used to detect LFC. The study mentioned above enrolled Chinese patients with T2DM, making it impossible to eliminate the confounding effect of the co-presence of liver steatosis and fibrosis. ${ }^{40}$ The present study is unique in that it recruited a normotensive and non-diabetic population, which can largely rule out these confounding

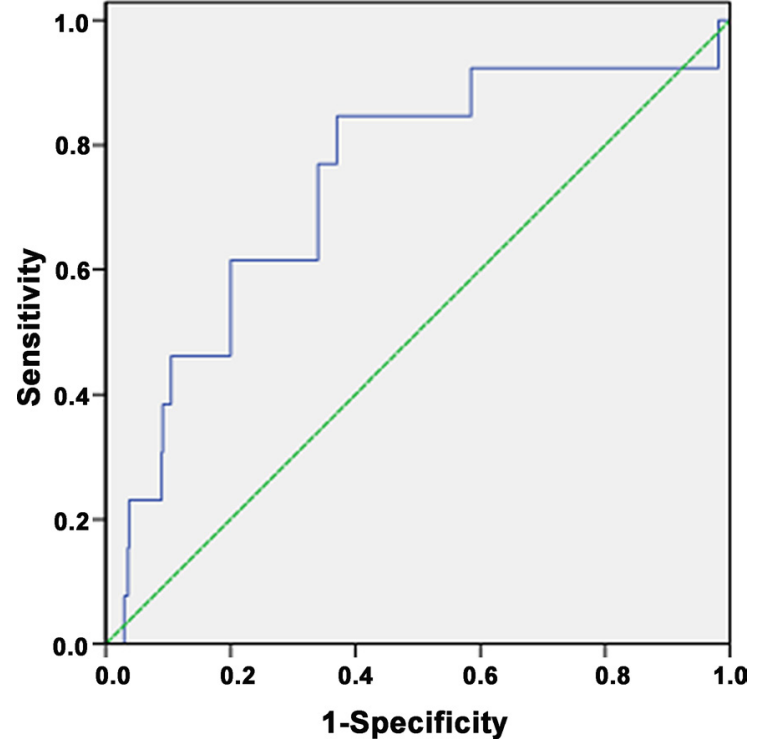

Figure 1 Receiver operating characteristic (ROC) curve evaluating the utility of liver fat content in the diagnosis of microalbuminuria (MA) in normotensive/euglycaemic middleaged and elderly people in Shanghai. The area under the ROC curve was 0.738 (95\% $\mathrm{Cl} 0.594$ to $0.882 ; \mathrm{p}=0.003)$.

factors. Moreover, our study used quantitative ultrasound to calculate LFC, which is more accurate than the ultrasound technique used in the previous study. The possible biological mechanisms underlying the effects of hepatic steatosis on MA development include reactive oxygen species, tumour necrosis factor-alpha, interleukin-6, plasminogen activator inhibitor-1 and other proinflammatory cytokines, which disrupt the glomerular endothelial glycocalyx and lead to MA. ${ }^{10}{ }^{41-43}$ Hepatokines, a group of functional proteins expressed by the liver are known to be involved in NAFLD. ${ }^{44}$ Future studies should examine these hepatokines in the patient population of the present study. Taken together, our findings suggest that LFC is strongly and independently associated with MA, implying that LFC can serve as a marker for early screening of MA in patients with suspected NAFLD.

The optimal LFC cut-off value for MA diagnosis was $6.82 \%$, a level suggestive of mildly fatty liver by histology. ${ }^{45}$ This result agrees with a more recent study indicating that NAFLD was associated with low-grade albuminuria, ${ }^{25}$ although the authors did not report an optimal LFC cutoff value for identifying MA. Taken at face value, our findings indicate that relatively small elevations of LFC may merit the evaluation of urinary albumin excretion rate in normotensive and non-diabetic people. Early evaluation of hepatic steatosis would facilitate the early detection of potential CKD, even for a relatively 'healthy' population. However, prospective studies are needed to validate our data and establish whether people with mildly elevated LFC are prone to CKD development and progression.

Interestingly, we failed to find associations of MA with BP or glucose level, contrary to reports in other populations. ${ }^{46} 47$ The lack of any associations of MA with BP or glucose level in our population may reflect the normal 
levels of FBG, PPG, SBP and DBP in our study participants. Moreover, eGFR, a classifying criterion for CKD, was not associated with LFC in this population; this absence of an association can potentially be explained by the very low prevalence of CKD in this relatively 'healthy' population and the limited sample size.

The multivariable models were adjusted either for WHR (models 2 and 4) and waist and hip circumferences (models 3 and 5) because the waist and hip circumferences are independent determinants of CVD risk and NAFLD. ${ }^{48}$ There were no significant changes in the results, suggesting that the effects of the waist and hip circumferences on MA are similar to WHR.

The present study has some limitations. First, because this was a cross-sectional study of the association between LFC and MA, causality cannot be inferred. Second, this was a single-centre study, so the generalisability of the findings is not known. Third, evaluation of hepatic steatosis was based on ultrasound and was not confirmed by liver biopsy. Although radiologic features cannot distinguish non-alcoholic steatohepatitis from other forms of NAFLD, routine liver biopsies were considered unacceptable for this epidemiologic study. Fourth, ACR in a morning urine sample was used as a surrogate marker for albumin excretion rate, and 24-hour urine collection was not performed. However, it is accepted that, under most circumstances, spot urine samples can be used to detect and monitor proteinuria in adults. ${ }^{35}$ Fifth, glycosylated haemoglobin is not included in the diagnostic criteria of diabetes in China because the test results are not standardised and consistent enough. Sixth, insulin levels were not measured. Because of the above limitations, prospective studies are needed to validate our findings.

\section{CONCLUSION}

In conclusion, LFC is an independent risk factor for MA in normotensive and non-diabetic middle-aged and elderly people. Even a small elevation in LFC is associated with an elevated risk of MA. These findings are of clinical relevance to the early detection of CKD risk in people with NAFLD.

\section{Author affiliations \\ ${ }^{1}$ Department of Endocrinology and Metabolism, Zhongshan Hospital Fudan University, Shanghai, China \\ ${ }^{2}$ Department of Geriatric Medicine, Fujian Provincial Hospital, Fujian Provincial Institute of Clinical Geriatrics, Fujian Provincial Key Laboratory of Geriatric Diseases, Fujian Medical University, Fuzhou, China \\ ${ }^{3}$ Department of Ultrasonography, Zhongshan Hospital Fudan University, Shanghai, China \\ ${ }^{4}$ Statistics, School of Public Health, Fudan University, Shanghai, China ${ }^{5}$ Department of Evidence Base Medicine Center, Zhongshan Hospital Fudan University, Shanghai, China}

Acknowledgements We are grateful to Professor Xin Gao from Zhongshan Hospital Fudan University for her supervision when pursuing my doctoral degree. We are grateful to Xunsheng Chen and Lin Shi from the Health Care Center of Changfeng Community, Shanghai, for their kind help at various stages of this study.

Contributors XL, MX, HM, YH, HY, HL, NZQ, JG and XG contributed to the conception and design of the study. XL, MX, HM and WH performed the data collection in the community. NZQ and JG analysed and interpreted the data. $X \mathrm{~L}$ and $X G$ wrote the manuscript with inputs from all the other authors. XG approved the final version of the manuscript before submission for publication. XG headed the project, supervised and conducted the study.

Funding This work was supported by grants from the National Key Technologies R\&D Program (Grant No. 2008 BAl52B03) to XG and (Grant No. 2009BAl80B01) to XG, National Key Technologies R\&D Program (Grant No. 2012CB524906) to XG, Science and Technology Commission of Shanghai Municipality (Grant No. 10411956400) to XG and (Grant No. 10411962600) to HY and National Basic Research Program of China (2011CB504004) (http://www.973.gov.cn/Default_3. aspx).

Competing interests None declared.

Patient consent for publication Not required.

Ethics approval The study protocol was approved by the Research Ethics Committee of Shanghai Health Bureau (2008-119). Written informed consent was obtained from all participants. Physical examinations, laboratory assessments and liver ultrasound scans were performed on the same morning for each individual at Changfeng Community Hospital.

Provenance and peer review Not commissioned; externally peer reviewed.

Data availability statement Data are available on reasonable request. The datasets used and/or analysed during the current study are available from the corresponding author on reasonable request.

Supplemental material This content has been supplied by the author(s). It has not been vetted by BMJ Publishing Group Limited (BMJ) and may not have been peer-reviewed. Any opinions or recommendations discussed are solely those of the author(s) and are not endorsed by BMJ. BMJ disclaims all liability and responsibility arising from any reliance placed on the content. Where the content includes any translated material, BMJ does not warrant the accuracy and reliability of the translations (including but not limited to local regulations, clinical guidelines, terminology, drug names and drug dosages), and is not responsible for any error and/or omissions arising from translation and adaptation or otherwise.

Open access This is an open access article distributed in accordance with the Creative Commons Attribution Non Commercial (CC BY-NC 4.0) license, which permits others to distribute, remix, adapt, build upon this work non-commercially, and license their derivative works on different terms, provided the original work is properly cited, appropriate credit is given, any changes made indicated, and the use is non-commercial. See: http://creativecommons.org/licenses/by-nc/4.0/.

ORCID iDs

Yu Hu http://orcid.org/0000-0003-3883-3887

Xin Gao http://orcid.org/0000-0003-1864-7796

\section{REFERENCES}

1 Kitade H, Chen G, Ni Y, et al. Nonalcoholic fatty liver disease and insulin resistance: new insights and potential new treatments. Nutrients 2017;9. doi:10.3390/nu9040387. [Epub ahead of print: 14 Apr 2017].

2 Younossi Z, Anstee QM, Marietti M, et al. Global burden of NAFLD and NASH: trends, predictions, risk factors and prevention. Nat Rev Gastroenterol Hepatol 2018;15:11-20.

3 Fan J-G, Kim S-U, Wong VW-S. New trends on obesity and NAFLD in Asia. $J$ Hepatol 2017;67:862-73.

4 Neuschwander-Tetri BA. Non-Alcoholic fatty liver disease. BMC Med 2017:15:45.

5 Katsiki N, Perez-Martinez P, Anagnostis P, et al. Is nonalcoholic fatty liver disease indeed the hepatic manifestation of metabolic syndrome? Curr Vasc Pharmacol 2018;16:219-27.

6 McCullough AJ. The clinical features, diagnosis and natural history of nonalcoholic fatty liver disease. Clin Liver Dis 2004;8:521-33.

7 Tapper EB, Loomba R. Nonalcoholic fatty liver disease, metabolic syndrome, and the fight that will define clinical practice for a generation of hepatologists. Hepatology 2018;67:1657-9.

8 Stefan N, Häring H-U, Cusi K. Non-Alcoholic fatty liver disease: causes, diagnosis, cardiometabolic consequences, and treatment strategies. Lancet Diabetes Endocrinol 2019;7:313-24.

9 Grander C, Grabherr F, Moschen AR, et al. Non-Alcoholic fatty liver disease: cause or effect of metabolic syndrome. Visc Med 2016;32:329-34.

10 Satchell SC, Tooke JE. What is the mechanism of microalbuminuria in diabetes: a role for the glomerular endothelium? Diabetologia 2008;51:714-25. 
11 Hogg RJ, Furth S, Lemley KV, et al. National kidney Foundation's kidney disease outcomes quality initiative clinical practice guidelines for chronic kidney disease in children and adolescents: evaluation, classification, and stratification. Pediatrics 2003;111:1416-21.

12 Grupper A, Schwartz D, Berliner S, et al. Normal-range albuminuria in healthy subjects increases over time in association with hypertension and metabolic outcomes. J Am Soc Hypertens 2018;12:759-67.

13 Romundstad S, Holmen J, Kvenild K, et al. Microalbuminuria and all-cause mortality in 2,089 apparently healthy individuals: a 4.4-year follow-up study. The Nord-Trøndelag health study (Hunt), Norway. Am J Kidney Dis 2003;42:466-73.

14 Klausen K, Borch-Johnsen K, Feldt-Rasmussen B, et al. Very low levels of microalbuminuria are associated with increased risk of coronary heart disease and death independently of renal function, hypertension, and diabetes. Circulation 2004;110:32-5.

15 Cirillo M, Senigalliesi L, Laurenzi M, et al. Microalbuminuria in nondiabetic adults: relation of blood pressure, body mass index, plasma cholesterol levels, and smoking: the Gubbio population study. Arch Intern Med 1998;158:1933-9.

16 de Jong PE, Curhan GC, Screening CGC. Screening, monitoring, and treatment of albuminuria: public health perspectives. J Am Soc Nephrol 2006;17:2120-6.

$17 \mathrm{Li} \mathrm{X}-\mathrm{H}$, Lin $\mathrm{H}-\mathrm{Y}$, Wang S-H, et al. Association of microalbuminuria with metabolic syndrome among aged population. Biomed Res Int 2016;2016:9241278.

18 Hwang ST, Cho YK, Yun JW, et al. Impact of non-alcoholic fatty liver disease on microalbuminuria in patients with prediabetes and diabetes. Intern Med J 2010;40:437-42.

19 Yilmaz Y, Alahdab YO, Yonal O, et al. Microalbuminuria in nondiabetic patients with nonalcoholic fatty liver disease: association with liver fibrosis. Metabolism 2010;59:1327-30.

20 Yeung M-W, Wong GL-H, Choi KC, et al. Advanced liver fibrosis but not steatosis is independently associated with albuminuria in Chinese patients with type 2 diabetes. J Hepatol 2017. doi:10.1016/j. jhep.2017.09.020. [Epub ahead of print: 06 Oct 2017] (published Online First: 2017/10/11).

21 Targher G, Chonchol MB, Byrne CD. Ckd and nonalcoholic fatty liver disease. Am J Kidney Dis 2014;64:638-52.

22 Targher G, Byrne CD. Non-Alcoholic fatty liver disease: an emerging driving force in chronic kidney disease. Nat Rev Nephrol 2017;13:297-310.

23 Mantovani A, Zaza G, Byrne CD, et al. Nonalcoholic fatty liver disease increases risk of incident chronic kidney disease: a systematic review and meta-analysis. Metabolism 2018;79:64-76.

24 Sun D-Q, Ye F-Z, Kani HT, et al. Higher liver stiffness scores are associated with early kidney dysfunction in patients with histologically proven non-cirrhotic NAFLD. Diabetes Metab 2020;46:288-95.

25 Lin L, Lu J, Huang X, et al. Nonalcoholic fatty liver disease is associated with low-grade albuminuria in Chinese adults (change not displayed). QJM 2016;109:737-43.

26 Targher G, Chonchol M, Bertolini L, et al. Increased risk of CKD among type 2 diabetics with nonalcoholic fatty liver disease. J Am Soc Nephrol 2008;19:1564-70.

27 Targher G, Mantovani A, Pichiri I, et al. Nonalcoholic fatty liver disease is independently associated with an increased incidence of chronic kidney disease in patients with type 1 diabetes. Diabetes Care 2014;37:1729-36.

28 Sun D-Q, Zheng Kl, Xu G, et al. Pnpla3 rs738409 is associated with renal glomerular and tubular injury in NAFLD patients with persistently normal ALT levels. Liver Int 2020;40:107-19.

29 Wijarnpreecha K, Thongprayoon C, Boonpheng B, et al. Nonalcoholic fatty liver disease and albuminuria: a systematic review and metaanalysis. Eur J Gastroenterol Hepatol 2018;30:986-94.
30 Gao X, Hofman A, Hu Y, et al. The Shanghai Changfeng study: a community-based prospective cohort study of chronic diseases among middle-aged and elderly: objectives and design. Eur $J$ Epidemiol 2010;25:885-93.

31 Chobanian AV, Bakris GL, Black HR, et al. Seventh report of the joint National Committee on prevention, detection, evaluation, and treatment of high blood pressure. Hypertension 2003;42:1206-52.

32 American Diabetes Association. Standards of medical care in diabetes-2018 abridged for primary care providers. Clin Diabetes 2018;36:14-37.

33 Alberti KGMM, Eckel RH, Grundy SM, et al. Harmonizing the metabolic syndrome: a joint interim statement of the International diabetes Federation Task force on epidemiology and prevention; National heart, lung, and blood Institute; American heart association; world heart Federation; international atherosclerosis Society; and international association for the study of obesity. Circulation 2009;120:1640-5.

34 Xia M-F, Yan H-M, He W-Y, et al. Standardized ultrasound hepatic/ renal ratio and hepatic attenuation rate to quantify liver fat content: an improvement method. Obesity 2012;20:444-52.

35 Levey AS, Coresh J, Balk E, et al. National kidney Foundation practice guidelines for chronic kidney disease: evaluation, classification, and stratification. Ann Intern Med 2003;139:137-47.

36 Levey AS, Stevens LA, Schmid $\mathrm{CH}$, et al. A new equation to estimate glomerular filtration rate. Ann Intern Med 2009;150:604-12.

37 Hofman A, Grobbee DE, de Jong PT, et al. Determinants of disease and disability in the elderly: the Rotterdam elderly study. Eur $J$ Epidemiol 1991;7:403-22.

38 Wong VW-S, Chu WC-W, Wong GL-H, et al. Prevalence of nonalcoholic fatty liver disease and advanced fibrosis in Hong Kong Chinese: a population study using proton-magnetic resonance spectroscopy and transient elastography. Gut 2012;61:409-15.

39 Stefan N, Häring H-U, Schulze MB. Metabolically healthy obesity: the low-hanging fruit in obesity treatment? Lancet Diabetes Endocrinol 2018;6:249-58

40 Aller de la Fuente R, Mora Cuadrado N, Tafur C, et al. Histopathological differences in patients with biopsy-proven non-alcoholic fatty liver disease with and without type 2 diabetes. Endocrinol Diabetes Nutr 2018;65:354-60.

41 Targher G, Bertolini L, Scala L, et al. Plasma PAI-1 levels are increased in patients with nonalcoholic steatohepatitis. Diabetes Care 2007:30:e31-2.

42 Targher G. Relationship between high-sensitivity C-reactive protein levels and liver histology in subjects with non-alcoholic fatty liver disease. J Hepatol 2006;45:879-81.

43 Targher G, Chonchol M, Zoppini G, et al. Risk of chronic kidney disease in patients with non-alcoholic fatty liver disease: is there a link? J Hepatol 2011;54:1020-9.

44 Artunc F, Schleicher E, Weigert C, et al. The impact of insulin resistance on the kidney and vasculature. Nat Rev Nephrol 2016;12:721-37.

45 McPherson S, Jonsson JR, Cowin GJ, et al. Magnetic resonance imaging and spectroscopy accurately estimate the severity of steatosis provided the stage of fibrosis is considered. $J$ Hepatol 2009;51:389-97.

46 Rosenbaum P, Gimeno SGA, Sanudo A, et al. Independent impact of glycemia and blood pressure in albuminuria on high-risk subjects for metabolic syndrome. Clin Nephrol 2004;61:369-76.

47 Palaniappan L, Carnethon M, Fortmann SP. Association between microalbuminuria and the metabolic syndrome: NHANES III. Am J Hypertens 2003;16:952-8.

48 Stefan N. Causes, consequences, and treatment of metabolically unhealthy fat distribution. Lancet Diabetes Endocrinol 2020;8:616-27. 